\title{
Validación de un protocolo fotográfico para la digitalización de muestras de herbario de especies tropicales
}

\author{
Validation of a photographic protocol for digitalization of herbarium samples of \\ tropical species
}

\section{Validação de um protocolo fotográfico para a digitalização de amostras de herbário de espécies tropicais}

\author{
Juan Carlos Valverde-Otálora ${ }^{10}$ \\ Ruperto Quesada ${ }^{2}$ \\ Casia Soto ${ }^{3}$ \\ Dagoberto $\mathrm{Arias}^{4}$ (i)
}

Recibido: septiembre 2019

Aceptado: abril 2020

Para citar este artículo: Valverde-Otálora, J.C., Quesada, R., Soto, C., Arias, D. (2020). Validación de un protocolo fotográfico para la digitalización de muestras de herbario de especies tropicales. Revista Científica, 38(2), 147-159. https://doi.org/10.14483/23448350.15362

\section{Resumen}

El estudio se validó de un protocolo fotográfico para la digitalización de muestras de herbario a partir de análisis de colorimetría digital. Para lo cual se utilizaron diez especies arbóreas en condición de herbario y se evaluó cinto tipos de velocidad de obturación, apertura de objetivo e ISO con fin identificar mejor ajuste y luego identificar el mejor protocolo fotográfico a partir de las variables $L^{*}, a^{*}, b^{*}$, diferencial de color $\left(\Delta E^{*}\right)$ y chroma $\left(\Delta C^{*}\right)$. Los resultados mostraron que las velocidades de obturación de 1/3", $1 / 5^{\prime \prime}$ y 1 " mostraron $\Delta E^{*}$ inferiores a 5 , considerados como visibles, la a apertura de objetivo f/ 5.6 y f/7.1 mostraron valores $\Delta E^{*}$ menores a 5,5 siendo mejor se adaptaron, mientras en ISO, los valores 100, 200 y 320 presentaron valores $\Delta E^{*}$ visibles y menores a 5,3 . En cuanto la determinación del mejor protocolo para las 10 especies fue CP1 con una velocidad de $1 / 3^{\prime \prime}$, con apertura de f/5.5 e ISO 100 que presentó un $\Delta E^{*}$ promedio de 4,1 considerado como visible y significativamente menor al resto de los tratamientos que variaron de 5,1 a 29,8, siendo cambios de visibles a totales de color y evidenciando con acumulaciones de color de 6 a $12\left(\Delta C^{*}\right)$. Finalmente, IRF mostró los usuarios percibieron que CP1 mostró mejor ajuste al material físico $(0,95)$ con respeto a los demás protocolos (promedio 0,71 ).

Palabras clave: apertura de objetivo, CIELab, dendrología, especies arbóreas, ISO, velocidad de obturación.

1. Instituto Tecnológico de Costa Rica, Laboratorio de ecofisiología forestal y aplicaciones Ecosistémicas (ECOPLANT), Cartago, Costa Rica. jcvalverde@tec.ac.cr

2. Instituto Tecnológico de Costa Rica, Escuela de Ingeniería Forestal, Cartago, Costa Rica. rquesada@tec.ac.cr

3. Instituto Tecnológico de Costa Rica, Escuela de Ingeniería Forestal, Cartago, Costa Rica. csoto@tec.ac.cr

4. Instituto Tecnológico de Costa Rica, Laboratorio de ecofisiología forestal y aplicaciones Ecosistémicas (ECOPLANT), Cartago, Costa Rica. darias@tec.ac.cr 


\section{Abstract}

The study was validated of a photographic protocol for the digitalization of herbarium samples from digital colorimetry analysis. For which ten tree species were used in herbarium condition and belt types of shutter speed, objective aperture and ISO were evaluated in order to identify better fit and then identify the best photographic protocol from the variables $L^{*}, a^{*}, b^{*}$, color differential $\left(\Delta \mathrm{E}^{*}\right)$ and chroma $\left(\Delta \mathrm{C}^{*}\right)$. The results showed that the shutter speeds of $1 / 3^{\prime \prime}, 1 / 5^{\prime \prime}$ and 1 "showed $\Delta E *$ less than 5 , considered as visible, the aperture of objective $f$ / 5.6 and $\mathrm{f} / 7.1$ showed $\Delta \mathrm{E} *$ values less than 5,5 being better adapted, while in ISO, values 100, 200 and 320 showed visible $\Delta \mathrm{E} *$ values and less than 5.3 . As soon as the determination of the best protocol for the 10 species was CP1 with a speed of $1 / 3$ ", with an aperture of $\mathrm{f} / 5.5$ and ISO 100 that presented an average $\Delta \mathrm{E} *$ of 4.1 considered as visible and significantly lower than the rest of treatments that varied from 5.1 to 29.8 , being changes from visible to total color and evidencing with color accumulations of 6 to $12(\Delta \mathrm{C} *)$. Finally, IRF showed the users perceived that CP1 showed a better fit to the physical material $(0.95)$ with respect to the other protocols (average 0.71 ).

Keywords: objective aperture, CIELab, dendrology, tree species, ISO, shutter speed.

\section{Resumo}

O estudo foi validado de um protocolo fotográfico para a digitalização de amostras de herbário a partir de análises de colorimetria digital. Para as quais dez espécies de árvores foram utilizadas na condição de herbário e os tipos de correia da velocidade do obturador, a abertura objetiva e a ISO foram avaliadas para identificar melhor ajuste e, em seguida, identificar o melhor protocolo fotográfico das variáveis $L^{*}, a *, b *$, diferencial de $\operatorname{cor}(\Delta \mathrm{E} *)$ e croma $\left(\Delta \mathrm{C}^{*}\right)$. Os resultados mostraram que as velocidades do obturador de 1/3", 1/5" e 1 "apresentaram $\Delta \mathrm{E} *$ menor que 5 , considerada visível, a abertura da objetiva f / 5.6 ef / 7.1 apresentou valores $\Delta E *$ inferiores a 55 sendo melhor adaptados, enquanto na ISO, os valores 100, 200 e 320 apresentaram valores visíveis de $\Delta \mathrm{E} *$ e menores que 5,3 . Assim que a determinação do melhor protocolo para as 10 espécies foi CP1 com velocidade de 1/3", com uma abertura de f / 5.5 e ISO 100 que apresentou um $\Delta \mathrm{E} *$ médio de 4,1 considerado como visível e significativamente menor que o restante tratamentos que variaram de 5,1 a 29,8, sendo alterações de visível para total e evidenciando acúmulos de 6 a $12(\Delta C *)$. Por fim, a IRF mostrou que os usuários perceberam que o CP1 apresentava melhor ajuste ao material físico $(0,95)$ em relação aos demais protocolos (média 0,71).

Palavras-chaves: abertura objetiva, CIELab, dendrologia, espécies arbóreas, ISO, velocidade do obturador.

\section{Introducción}

La región tropical se ha caracterizado por presentar una amplia diversidad de especies arbóreas (WiIlis et al., 2017), aspecto que ha incidido que en los últimos 150 años se hayan realizado una gran cantidad de expediciones científicas enfocadas en colectas botánicas que permitieron ubicar, recolectar, procesar e identificar las especies existentes en los distintos bosques tropicales (Hutchens, 2011) y generando con ello amplias colecciones botánicas que incidieron en la creación de los herbarios (Shotbolta, Bükerb, y Ashmore, 2007). Las colecciones de muestras botánicas históricamente se han caracterizado por ser material en condición seco, representativo de cada especie, que permita entender la forma, dimensiones y características de la hoja, además, de su posición a nivel de rama o tallo y con preferencia en estado fértil (presencia de flores y frutos) (Riera, Sangil, y Sansón, 2015).

Las colecciones de herbario han permitido ser una fuente de conocimiento para la formación de expertos en identificación de especies (Magdalena, et al. 2018), para la documentación de usos económicos (Kao, Djendoel Soejarto, Kinghornc, y Oberlies, 2018) e históricos de las especies en una región (Blagoderov, Kitching, Livermore, Simonsen, y Smith, 2012). Las colecciones también han permitido ubicar espacialmente las áreas en las que se localizan poblaciones de cada especie (Martin, Quiroz-Claros, Brushb, y Zimmer, 2018), analizar las variaciones morfológicas de una especie según 
su procedencia (Rivers et al., 2011), ser un reporte histórico composición florísticas de distintas regiones tropicales y de entendimiento del cambio de la dinámica boscosa (Hutchens, 2011) y registro fenológico de cada especie (Magdalena et al., 2018). Rivers et al. (2011) mencionan que las colecciones botánicas permiten en primera instancia entender la dinámica del bosque tropical en cuanto composición y riqueza de especies, entender las épocas de mayor florescencia y el grado de salud que puede presentar una especie o una región (Shotbolta et al., 2007).

Sin embargo, en la actualidad las colecciones botánicas de herbarios han tenido un retardo tecnológico, el cual ha limitado su internacionalización, se continúa la estructura tradicional a ser utilizadas únicamente en un espacio físico con condiciones controladas y con un acceso único a la muestra físico, lo que ha limitado su consulta (van der Ent et al., 2019); por lo que en la última década se ha iniciado un proceso de digitalización de las muestras de herbario para la creación de herbarios digitales (Magdalena et al. 2018), como sitios de acceso de consulta y estudio de especies, lo cual ha posibilitado el análisis, comparación y entendimiento tanto de la variedad de una especie presente en varias regiones del planeta, como la variación de los ecosistemas tropicales (Unger, Merhof y Renner, 2016).

En el proceso de digitalización de muestras de herbario se deben considerar tres elementos fundamentales según mencionan Shotbolta et al. (2007): i. la versión digital de la muestra debe preservar las características de dimensionalidad, estabilidad y colorimetría de la muestra física, ii. la manipulación de las propiedades de la muestra deben ser mínimo, con ello evitar que el usuario que consulte genere entendimientos erróneos por procesamiento erróneo del material y iii. se debe respetar u mantener toda la información complementaria presente en la muestra, para lo cual se debe considerar la ficha de metadatos de las muestras y anotaciones existentes en el material. Conforme mayor sea la similitud entre las propiedades visuales presentes en la muestra física y digital, el carácter científico y representatividad del herbario digital será mayor (van der Entet al., 2019). Una de las propiedades en las que se debe tener mayor exactitud en el proceso de digitalización es el manejo del color (Park, 2012), debido que es muy susceptible a las condiciones ambientales en las que se capture, una reproducción digital de baja calidad o realizada sin un protocolo o procedimiento claro generaría distorsiones en el producto final, por lo que Kovtonyuk y Gatilova (2017) recomienda en este tipo de procedimientos de digitalización disponer de un protocolo claro, conciso y simple que sea capaz de ser reproducido por cualquier usuario.

En los procesos de digitalización de herbarios se han implementado dos líneas de instrumentalización: escaneo y fotografía (Thiers, Tulig y Watson, 2016). El primero se caracteriza por ser un procesamiento de alta calidad en el que se reproduce hasta el mínimo detalle de la muestra y en algunos casos el escaneo puede ser tridimensional (3D), sin embargo, presenta limitaciones ya que se requieren escáner de alta resolución y dimensiones mayores a los disponibles en el mercado, lo cual genera que su costo sea mayor, además que el procedimiento requiere un mayor tiempo por muestra (Barber, Lafferty y Landrum, 2013) Con la fotografía digital, se puede implementar un sistema de medición rápido, adaptable a las dimensiones de la muestra y con un coste menor, pero en el cual se debe tener un protocolo normalizado en cuanto conservación de las dimensiones de la fotografía, calidad de muestra y considerar aspectos controlados como la luminosidad, apertura de lente, velocidad de toma fotográfica, fondo y escala de la muestra.

En la fotografía de herbarios una de las variables que se debe controlar es el color, debido a que es muy susceptible a las condiciones de calibración de la cámara y condiciones del sitio (Drinkwater, Cubey y Haston, 2014), por lo que el desarrollo de protocolos que mantengan la mayor fidelidad de dicha propiedad es fundamental 
para la representatividad de la fotografía (Haston, Cubey, Pullan, Atkins y Harris, 2012). Para un mejor control es necesaria la utilización de análisis colorimétricos en los que se utilicen patrones de color como el CIELAB, que permiten cuantificar y dimensionar el grado de precisión del protocolo y los factores de corrección que permitan que las fotos tengan una variación mínima con la muestra física. De ahí que se planteó como objetivo validar de un protocolo fotográfico para la digitalización de muestras de herbario a partir de análisis de colorimetría digital. Con ello se pretende disponer de un protocolo funcional que permita generar imágenes colorimétricamente representativas de las muestras físicas de herbario y con ello el usuario disponga de un material representativo.

\section{Metodología}

\section{Especies evaluadas}

Se utilizaron diez especies arbóreas de Costa Rica (tabla 1), las muestras se caracterizaron por ser láminas de herbario en condición seca y procedentes de distintas áreas geográficas de Costa Rica y recolectadas entre el 1990 y 2016. El material formó parte del herbario de la Escuela de Ingeniería Forestal del Tecnológico de Costa Rica $\left(9,82^{\circ} \mathrm{N}, 83,91^{\circ} \mathrm{O}\right)$, el material se almacenó en muebles metálicos acondicionados a una temperatura promedio de $20{ }^{\circ} \mathrm{C}$ y humedad relativa promedio del $50 \%$, las especies seleccionadas se caracterizaron por tener una cantidad de láminas mayor a seis muestras, con distintas procedencias y épocas recolectadas, además de ser material el cual presentara claramente el haz y el envés de las hojas (mínimo 2 hojas con cada perspectiva) y ausencia de daños o defectos tanto del material secado, como de la lámina estudiada (que la lámina no presentara perforaciones, rayaduras, arrugas o defectos que dañaran la calidad de la muestra).

\section{Protocolo base de fotografía}

Previo al fotografiado, cada muestra se limpió con una brocha para eliminar el polvo, desechos o partículas que pudieran disminuir la calidad de la muestra. Las fotografías se generaron bajo en una misma superficie plana con fondo negro, la cámara se colocó a una altura de $60 \mathrm{~cm}$ sobre la muestra y se ubicaron tres lámparas LED (con una potencia de 40 Watts), a $20 \mathrm{~cm}$ alrededor de la muestra. La luminosidad del área fue contante y en promedio de 800 lux. Cada muestra se colocó en posición vertical y se le agregó una escala para dimensionar el tamaño de las muestras y

Tabla 1. Especies y cantidad de láminas implementadas para la validación colorimétrica de protocolo fotográfico para la virtualización de herbario en Costa Rica.

\begin{tabular}{ccc}
\hline Familia & Nombre científico & Cantidad de láminas \\
\hline Bignoniaceae & Tabebuia rosea (Bertol.) DC. & 8 \\
Burseraceae & Protium panamense (Rose) I.M.johnst. & 8 \\
Cannabaceae & Trema micrantha (L.) Blume & 7 \\
Clethraceae & Clethra mexicana DC. & 9 \\
Euphorbiaceae & Croton niveus Jacq. & 8 \\
Fabaceae / Mimosaceae & Cojoba costaricensis Britton y Rose & 8 \\
Fabaceae / Mimosaceae & Zygia longifolia (Humb. y Bonpl. ex Willd.) Britton y Rose & 9 \\
Fagaceae & Quercus insignis M. Martens y Galeotti & 7 \\
Lauraceae & Nectandra reticulata (Ruiz y Pav.) Mez & 10 \\
Meliaceae & Cedrela odorata L. & 6 \\
\hline
\end{tabular}

Fuente: Los autores. 
tener colores de referencia (color rojo, verde, azul y negro). Para todas las pruebas de fotografiado se utilizó una cámara Sony modelo Alpha 7 ILCE7K/B, la dimensión de la fotografía fue de $4000 x$ 6000 px (resolución de 24 MP) con un lente intercambiable de FE 3.5-5.6/28-70. Las fotografías se almacenaron en formato JPG y RAW.

\section{Variables evaluadas en el proceso fotográfico}

La cámara se usó en modo manual y se modificaron tres variables de exposición: velocidad de obturación (tiempo que se tarda en realizar la fotografía), apertura del objetivo (apertura del diafragma) y sensibilidad ISO (sensibilidad que tiene el sensor para captar luz). El estudio efectuó cuatro experimentos para determinar el ajuste fotográfico óptimo, a continuación, se explican:

Selección de velocidad de obturación. Se evaluaron cinco velocidades: 1/3", 1/5", 1", 2", 4"; todas las velocidades se probaron con la cámara en modo "S" (prioridad velocidad exposición) una apertura del objeto de f/5.6 e ISO de 320 según, esto se trabajó bajo la recomendación de Lang et al., (2017) para la estandarización de protocolos fotográficos.

Selección apertura del objetivo. Se implementaron cinco aperturas: $\mathrm{f} / 2.8, \mathrm{f} / 5.6, \mathrm{f} / 7.1, \mathrm{f} / 13$ y f/16. Las aperturas se probaron bajo el modo " $A$ " (prioridad apertura), en la que se evaluaron con una misma velocidad de obturación de 1/3" e ISO de 320 según recomienda Lang et al., (2017) para la estandarización de protocolos fotográficos.
Selección del ISO óptimo. Se manejaron cinco valores de ISO: 100, 200, 320, 400 y 800 . Se mantuvo constante la velocidad de 1/3" y apertura de 5,6 para lo cual se implementó la cámara en modo manual " $M$ ", según menciona Lang et al., (2017) para validación de protocolos fotográficos.

A partir de los resultados previos de los experimentos anteriores se plantearon seis posibles combinaciones con potencialidad (CP) para generar representatividad fotográfica de las muestras (tabla 2).

\section{Análisis de color}

El color se evaluó tanto en la muestra física como la imagen fotografiada con cada CP. Con las muestras físicas, la medición del color se realizó con un espectrofotómetro NIX Pro de cromatografía CIE estandarizado. El color se determinó entre el rango de los 400 a $700 \mathrm{~nm}$ con un puerto de medición de $10 \mathrm{~mm}$ de diámetro. Para la observación de la reflexión del componente especular (SCI modo) se incluyó en un ángulo de 10, que es normal para la superficie de hojas (D65 / 10); y con un D65 (correspondiente a la luz del día en $6500 \mathrm{~K}$ ), en cada muestra se midió el color en todas las hojas presentes en la muestra y se analizó tanto en el haz como envés, en cada hoja se recolectaron tres mediciones. En el caso de las fotografías, el color se determinó con el programa Adobe Photoshop CC2019, se implementó la herramienta cuentagotas y se analizó por cada hoja 25 mediciones.

Tabla 2. Combinaciones con potencialidad (CP) de representatividad fotográfica para ser empleadas en muestras secas de diez especies forestales en condiciones de herbario en Costa Rica.

\begin{tabular}{cccc}
\hline Código de protocolo & Velocidad de obturación & Apertura del objetivo & ISO \\
\hline CP1 & $1 / 3^{\prime \prime}$ & $\mathrm{F} 5.6$ & 100 \\
CP2 & $1^{\prime \prime}$ & $\mathrm{F} 7.1$ & 200 \\
CP3 & $1 / 5^{\prime \prime}$ & $\mathrm{F} 5.6$ & 320 \\
CP4 & $1 / 3^{\prime \prime}$ & $\mathrm{F} 5.6$ & 200 \\
CP5 & $1^{\prime \prime}$ & $\mathrm{F} 7.1$ & 100 \\
CP6 & $1 / 5^{\prime \prime}$ & $\mathrm{F} 7.1$ & 320 \\
\hline
\end{tabular}

Fuente: Los autores. 
El color se evaluó en formato CIELAB, el cual generó tres parámetros para explicar el color que consistieron en: $L^{*}$ (luminosidad), a * (tendencia de color de rojo a verde) y $b *$ (tendencia de color de amarillo a azul).

\section{Diferencial del color}

El diferencial de color $\left(\Delta E^{*}\right)$ se definió como la variación neta del color entre dos escenarios, según lo establecido por la norma ASTM D 2244 (2013) cuya fórmula es detallada en la ecuación 1. El $\Delta E^{*}$ se implementó para encontrar diferencias el color entre las fotografías y muestras físicas y con ello identificar el grado de representatividad del color utilizando las categorías de (Cui, Kamdem y Rypstra, 2004), que establece cinco niveles de cambio de color percibido por el ojo humano: si $\left.0<\Delta E^{*}\right\rangle$ 0,5 , el color el cambio no es percibido por el ojo humano; si $1,5<\Delta E^{*}>3,0$, el cambio de color apenas se percibe; si $3,0<\Delta E^{*}>6,0$, se percibe el cambio; si $6,0<\Delta E^{*}>12,0$, el cambio de color es bastante notable por el ojo humano y finalmente si $\Delta E^{*}$ es mayor que 12 , hay un cambio total de color.

$$
\Delta E^{*}=\sqrt{\left(\Delta L^{*}\right)^{2}+\left(\Delta a^{*}\right)^{2}+\left(\Delta b^{*}\right)^{2}}
$$

Donde: $\Delta E^{*}$ es el diferencial del color; $\Delta L^{*}$ es $L^{*}$ del protocolo de fotografía analizado - $L^{*}$ muestra física; $\Delta a^{*}$ es a* del protocolo de fotografía analizado - a* muestra física; $\Delta b^{*}$ es $\mathrm{b}^{*}$ del protocolo de fotografía analizado - $b^{*}$ muestra física.

\section{Diferencial chroma}

Se evaluó el diferencial chroma $\left(\Delta C^{*}\right)$ se definió como la variación neta de la saturación del color (a* y b*) entre dos escenarios, según lo establecido por la norma ASTM D 2244 (ASTM, 2013) cuya fórmula es detallado en la ecuación 2. Este parámetro permite estimar si hay pérdida o ganancia de color indiferentemente de la luminosidad.

$$
\Delta E^{*}=\sqrt{\left(\Delta a^{*}\right)^{2}+\left(\Delta b^{*}\right)^{2}}
$$

Donde: $\Delta C^{*}$ es el diferencial del chroma; $\Delta a^{*}$ es $\mathrm{a}^{*}$ del protocolo de fotografía analizado - $\mathrm{a}^{*}$ muestra física; $\Delta b^{*}$ es $b^{*}$ del protocolo de fotografía analizado - $b^{*}$ muestra física.

\section{Índice representatividad colorimétrica}

Se desarrolló un índice de sensibilidad colorimétrica (IRC), para evaluar el efecto de la calidad fotográfica obtenido con cada protocolo y compararlo con respeto a los análisis cuantitativo-colorimétricos. Para lo cual se utilizaron 20 personas con conocimiento en identificación de especies botánicas y se les mostró tanto la muestra física como las fotografías (figura 1) de los seis CP. Para ello se evaluó: i. similitud de color (que tanto varió el color de la muestra con las fotos), ii. armonía del color (los colores en conjunto tienen carencia o lógica con respecto al material) y iii. efecto del color en la identificación de la muestra (la variación del color evita notar características de la especie). Cada variable se evaluó con una escala el 1 al 10 donde 1 fue el valor mínimo y 10 el máximo; cada variable se utilizó en la ecuación 3, la cual presenta una escala del 0 al 10 donde 0 es el valor mínimo que indica que el IRC es bajo y 10 indica es alto.

$$
I R C=\frac{(S C * 0,33)+(A R * 0,33)+(E M C * 0,33)}{10}
$$

Donde: IRC es el índice de representatividad del color; SC es la similitud del color, Ar es la armonía del color y EMC es el entendimiento de la muestra por el color.

\section{Diseño experimental y análisis de resultados}

Primeramente, se generó una caracterización colorimétrica de las muestras físicas con los parámetros $L^{*}, a^{*}$ y $b^{*}$ con el fin de identificar variaciones de color entre las submuestras de cada muestra y a su vez establecer las diferencias estadísticas entre colores del haz y envés, para lo cual se realizó un análisis de varianza (ANDEVA de una vía), una validación de los supuestos y en caso de determinar 

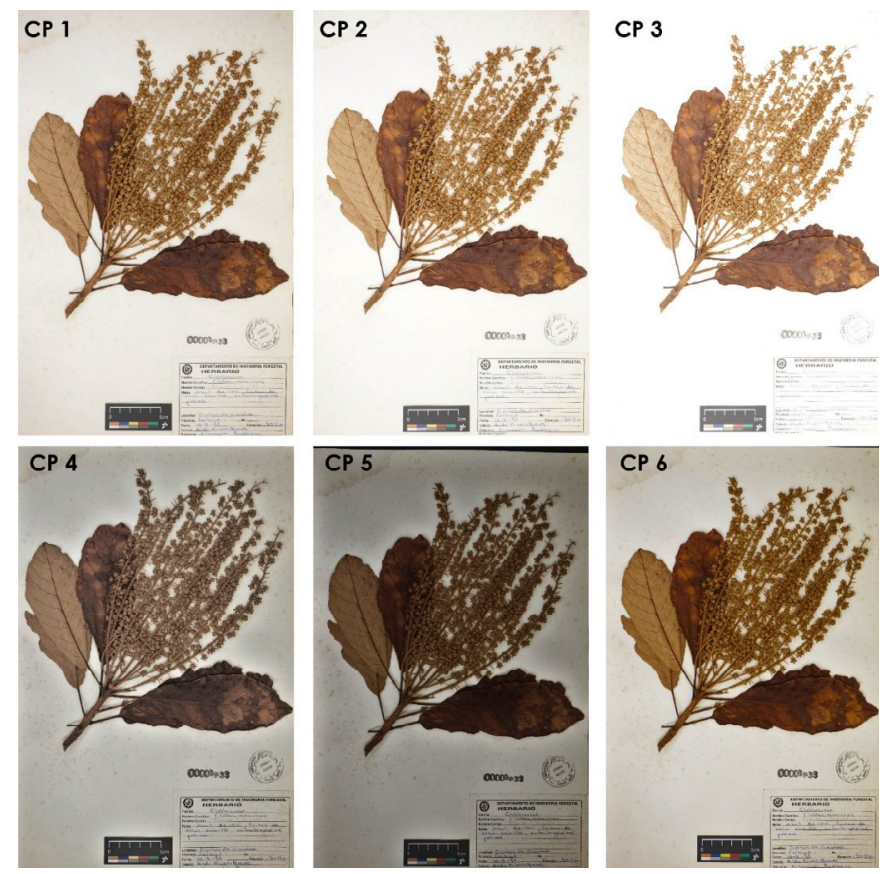

Figura 1. Muestra fotográfica de Cletha mexicana analizada-con los seis potenciales protocolos implementados en el proceso de digitación de especies arbóreas en condiciones de herbario en Costa Rica.

Fuente: Los autores.

un efecto significativo se aplicó una prueba de Tukey para identificar las diferencias.

Posteriormente, se analizó la diferencia entre los valores de color de la muestra física con respecto a los distintos protocolos de velocidad del obturador, apertura de objetivo e ISO, con el fin de identificar los valores que generaron menor diferencial del color y con ello la valoración de las seis combinaciones de CP, para cual se aplicó nuevamente un ANDEVA con las condiciones anteriormente descritas.

Todos los análisis se realizaron con una significancia de 0,05 y se utilizó el programa STATISTICA 9.1.

\section{Resultados}

\section{Caracterización inicial del material}

Se encontraron distintos patrones de coloración entre el haz y envés de las hojas de las diez especies analizadas. Para T. Rosea, C. mexicana y C. niveus no se encontraron diferencias significativas en los valores $L^{*}, a^{*}$ y $b^{*}$ entre el haz y envés, encontrando una coloración en $\mathrm{L}^{*}$ considerada como moderadamente grisácea (varió de 58,45 a 69,44), con a* la moderadamente rojiza y con una variación de 8,04 a 15,16 y con b* la coloración tendió a ser amarillenta con valores de 14,56 a 33,56. En el caso de Z. longifolium, Q. insignis y N. reticulata se dio el mismo comportamiento, con los valores $a^{*} y b^{*}$ no presentaron cambios entre haz y envés, presentando un patrón de color rojizo amarillento, con L* se dio un oscurecimiento del color en el envés que decrecieron en promedio de 55,80 a 44,43. Con $P$. panamense y $C$. odorata el envés presentó una disminución de la luminosidad $(45,26)$ con respecto al haz $(56,3)$; sin embargo el envés presentó una coloración rojizo amarillenta significativamente mayor al haz (en a* aumentó un 35,5 \% y en b* un 10,3 $\%)$. Por su parte T. micrantha mostró incrementos del 10 al 32,4\% de los tres parámetros del color en el envés con respecto al haz. Finalmente, con $C$. arborea los valores de $L^{*}$ y $b^{*}$ decrecieron un 22,4 $\%$ en el envés con respecto al haz, mientras $b^{*}$ no mostró variaciones significativas. 
Tabla 3. Valores de color $\mathrm{L}^{*}, \mathrm{a}^{*}$ y $\mathrm{b}^{*}$ obtenidos en forma directa en el haz y envés de diez especies arbóreas preservadas en condiciones de herbario.

\begin{tabular}{|c|c|c|c|c|c|c|}
\hline \multirow{2}{*}{ Especie } & \multicolumn{3}{|c|}{ Haz } & \multicolumn{3}{|c|}{ Envés } \\
\hline & $\mathbf{L}^{*}$ & $\mathrm{a}^{*}$ & $\mathbf{b}^{*}$ & $\mathbf{L}^{*}$ & $\mathrm{a}^{*}$ & $\mathbf{b}^{*}$ \\
\hline T. rosea & $69,44 \mathrm{a}(6.14)$ & $8,04 \mathrm{a}(5,11)$ & $14,56 \mathrm{a}(5,3)$ & $65,44 \mathrm{a}(7,02)$ & $10,55 \mathrm{a}(8,45)$ & $15,88 \mathrm{a}(7,56)$ \\
\hline P. panamense & $52,52 \mathrm{a}(4,55)$ & $13,22 \mathrm{a}(7,56)$ & $26,24 \mathrm{a}(8,99)$ & $40,11 \mathrm{a}(7,22)$ & $17,19 b(3,44)$ & $19,11 b(4,03)$ \\
\hline T. micrantha & $50,11 \mathrm{a}(4,55)$ & $10,77 \mathrm{a}(3,59)$ & $20,14 \mathrm{a}(4,00)$ & $54,11 b(3.98)$ & $30,14 a(5,79)$ & $29,45 b(6,08)$ \\
\hline C. mexicana & $58,45 \mathrm{a}(5,78)$ & $15,16 \mathrm{a}(3,88)$ & $24,24 \mathrm{a}(4,56)$ & $58,34 \mathrm{a}(4,59)$ & $14,11 \mathrm{a}(6,01)$ & $20,22 \mathrm{a}(5,16)$ \\
\hline C. niveus & $59,41 \mathrm{a}(7,01)$ & $13,02 \mathrm{a}(4,99)$ & $31,07 \mathrm{a}(8,89)$ & $58,22 \mathrm{a}(7,56)$ & $14,78 \mathrm{a}(4,16)$ & $33,56 \mathrm{a}(5,07)$ \\
\hline C. costaricensis & $49,11 \mathrm{a}(6,16)$ & $12,13 \mathrm{a}(4,01)$ & $28,16 \mathrm{a}(4,79)$ & $40,16 b(3,50)$ & $13,81 \mathrm{a}(4,00)$ & $30,11 b(7,13)$ \\
\hline Z. longifolia & $51,11 \mathrm{a}(5,41)$ & $13,88 \mathrm{a}(5,02)$ & $21,09 \mathrm{a}(9,11)$ & $40,05 b(8,56)$ & $14,91 \mathrm{a}(6,60)$ & $20,57 \mathrm{a}(6,07)$ \\
\hline Q. insignis & $54,05 a(5,99)$ & $13,44 \mathrm{a}(8,47)$ & $30,07 \mathrm{a}(8,14)$ & $47,22 b(9,54)$ & $15,6 \mathrm{a}(17,56)$ & $28,79 a(8,56)$ \\
\hline N. reticulata & $62,24 \mathrm{a}(4,55)$ & $19,4 a(5,55)$ & $29,77 a(6,01)$ & $46,01 \mathrm{~b}(7,00)$ & 18,01a (3.98) & $27,56 a(5,49)$ \\
\hline C. odorata & $60,11 \mathrm{a}(4,12)$ & $10,01 \mathrm{a}(3,77)$ & $27,56 \mathrm{a}(5,89)$ & $50,42 b(5,46)$ & $16,60 b(5,78)$ & $33,11 \mathrm{~b}(6,16)$ \\
\hline
\end{tabular}

Letras diferentes muestran diferencias significativas con a 0,05 .

Fuente: Los autores

\section{Análisis de las variables fotográficas probadas}

En la figura 2 se presentan los valores del $\Delta E^{*}$ para las variables de velocidad de obturador, apertura de objetivo e ISO. Para el factor de velocidad de obturador (figura 2a), se encontró un patrón similar para las diez especies, encontrando dos agrupaciones de tratamientos, las aperturas rápidas de 1/3", $1 / 5^{\prime \prime}$ y 1 " no presentaron diferencias entre sí y mostraron los $\Delta E^{*}$ significativamente menores (de 2,9 a $6,9)$, considerados como un cambio perceptible. El segundo grupo lo conformó las aperturas de 2 " y 4 " con $\Delta E^{*}$ significativamente mayores (de 8,7 a 19,6), considerados como un cambio total del color.

Con la apertura del objetivo (figura $2 b$ ), se encontró que $\mathrm{f} / 5.6$ y f/7.1 presentaron valores $\Delta E^{*}$ óptimos (de 2,8 a 6,1 ) considerados como perceptibles y significativamente menores a f/2.8, f/13 y f/16 que mostraron cambios totales del color $\left(\Delta E^{*}\right.$ de 8,6 a 23,5$)$ y mostraron una eficiencia debida al cambio radical de la coloración del material, en especial el aumento de la luminosidad ( $L^{*}$ hasta en un $42 \%$ ). Finalmente, con el ISO (figura 2c), el ISO 100, 200 y 320 no mostraron diferencias entre sí y obtuvieron un $\Delta E^{*}$ promedio de 4,8 considerado como un cambio perceptible; mientras el ISO 400 y 800 el $\Delta E^{*}$ promedio fue de 11,9 y es considerado como un cambio total del color que fue significativamente superior.

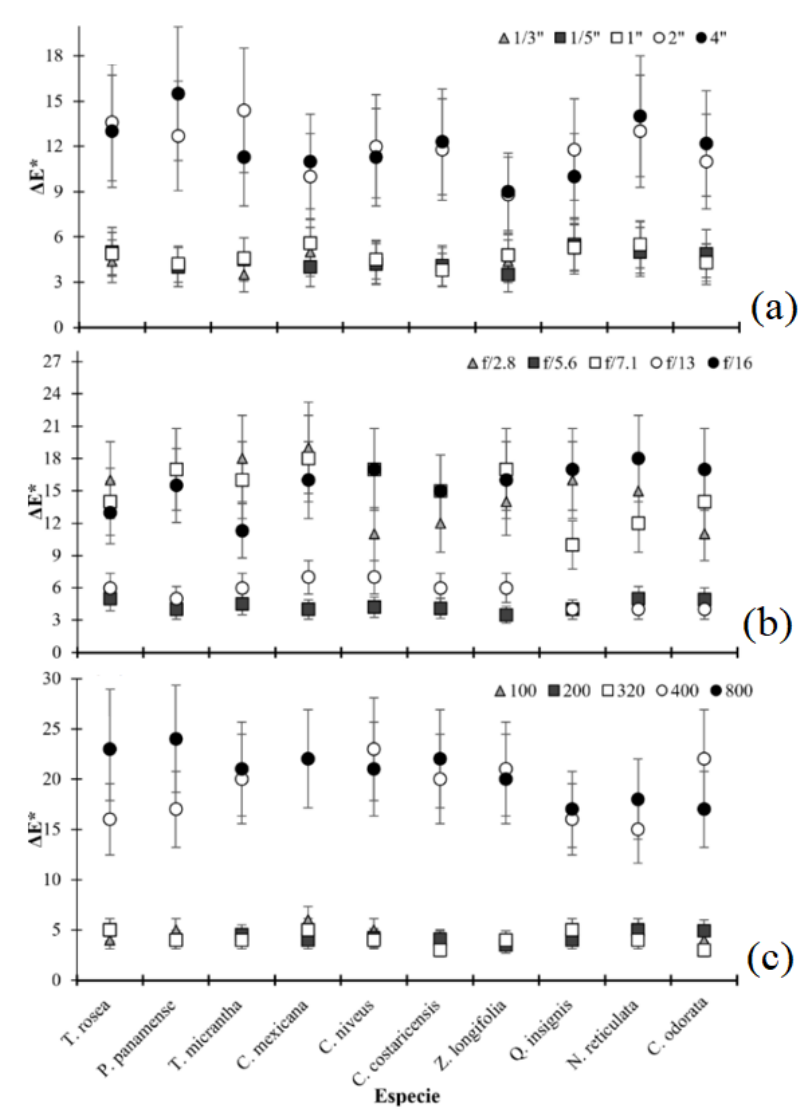

Figura 2. Valores de diferencial de color $\left(\Delta E^{*}\right)$ obtenidos a partir de la variación de velocidad de obturador (a), apertura de objetivo (b) e ISO (c) implementados en el proceso de digitalización de diez especies arbóreas en condiciones de herbario en Costa Rica.

Fuente: Los autores. 


\section{Determinación del mejor ajuste fotográfico}

Se obtuvo que únicamente el CP1 mostró uniformidad de resultados en las diez especies, los otros 5 protocolos mostraron variaciones significativas según la especie (figura 3a). CP1 mostró un valor $\Delta E^{*}$ promedio de 3,6 considerado como perceptible y su variación fue la menor de los protocolos evaluados; los restantes protocolos mostraron variaciones de 4,1 a 30,3 mostrando poca homogeneidad y estabilidad entre especies, lo cual se notó con variaciones significativas del color. Los protocolos CP5 y CP6 fueron los que presentaron mayor cambio del color y que incidió en la mayor ineficiencia (promedio $\Delta E^{*} 22,4 \pm 9,49$ ), en cambio CP2 mostró una adaptación parcial (a cinco de las especies implementadas) con valores de $\Delta E^{*}$ inferiores a 5,4 pero significativamente mayores a CP1.

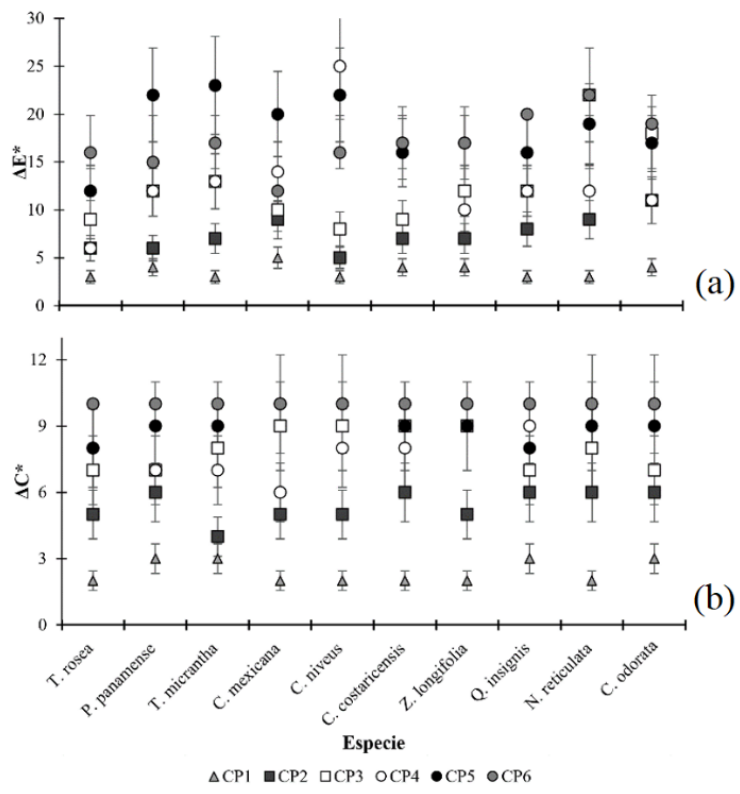

Figura 3. Valores de diferencial de color $\left(\Delta E^{*}\right)$ (a) y diferencial de Chroma $\left(\Delta C^{*}\right)$ (b) obtenidos a partir de los seis CP implementados en el proceso de digitalización de diez especies arbóreas en condiciones de herbario en Costa Rica.

Fuente: Los autores.

En cuanto el $\Delta C^{*}$ (figura $3 \mathrm{~b}$ ), se mantuvo la tendencia de que la menor ganancia del color fue
CP1, la cual fue menor a 4,3 considerada significativamente baja con respecto a los otros cinco protocolos que variaron de 4,4 a 12,6 siendo CP6 el que mostró el mayor aumento de coloración de las muestras por lo que se puede decir que el CP1 fue el protocolo con mejor adaptación a las condiciones de fotografía empleadas.

Con el IRC (Tabla 4), el único protocolo que presentó homogeneidad entre las diez especies fue el PC1 que mostró los mayores índices (promedio 0,96), que fue significativamente mayor a los restantes protocolos que presentaron valores de 0,55 a 0,71 y que variaron significativamente en cada especie. En el análisis desarrollado de la percepción de la armonía, la coherencia del color es muy baja, la percepción de los usuarios después de aplicar el CP2 al CP6, es que los colores no guardan similitud con el material físico y con ello se reduce la capacidad de usar la imagen como referencia de identificación.

\section{Discusión}

\section{Diferenciación color entre haz y envés de las muestras y su efecto en la digitación}

La variación colorimétrica encontrada entre el haz y el envés mostró variaciones debidas a aspectos como la composición química, presencia de ceras y taninos que interfieren en los procesos de oxidación y degradación de enzimas que se originan durante el proceso de secado y almacenado de las muestras (Souza y Hawkings, 2017). Estas variaciones resultan en una afectación de la homogeneidad del color (Haston, Cubey et al. 2012). La variación en especial de la luminosidad $\left(\mathrm{L}^{*}\right)$ se debe a la textura y grosor de la hoja, (Thiers et al., 2016) mencionan que el envés de las hojas tiende a aumentar la claridad del color conforme el grosor de la lámina sea mayor, esto debido que en el haz se desarrolla fotosíntesis, lo cual origina una coloración fuerte en especial de los valores $a^{*} y$ $b^{*}$ y una luminosidad baja con respecto al envés, pero en el proceso de secado y almacenamiento 
Tabla 4. Valores de IRC obtenidos para diez especies arbóreas preservadas en condiciones de herbario.

\begin{tabular}{|c|c|c|c|c|c|c|}
\hline Especie & CP1 & CP2 & CP3 & CP4 & CP5 & CP6 \\
\hline T. rosea & $0,99 \mathrm{a}(0,01)$ & $0,69 \mathrm{~b}(0,06)$ & $0,71 b(0,07)$ & $0,68 b(0,06)$ & $0,66 b(0,05)$ & $0,69 \mathrm{~b}(0,07)$ \\
\hline P. panamense & $0,95 \mathrm{a}(0,01)$ & $0,65 b(0,04)$ & $0,68 b(0,08)$ & $0,66 b(0,05)$ & $0,65 b(0,06)$ & $0,67 b(0,06)$ \\
\hline T. micrantha & $0,96 \mathrm{a}(0,01)$ & $0,67 b(0,04)$ & $0,69 \mathrm{~b}(0,06)$ & $0,66 b(0,07)$ & $0,66 \mathrm{~b}(0,06)$ & $0,69 \mathrm{~b}(0,07)$ \\
\hline C. mexicana & $0,97 \mathrm{a}(0,02)$ & $0,77 b(0,02)$ & $0,70 \mathrm{~b}(0,07)$ & $0,67 b(0,05)$ & $0,67 b(0,02)$ & $0,67 b(0,08)$ \\
\hline C. niveus & $0,95 \mathrm{a}(0,01)$ & $0,65 b(0,05)$ & $0,66 b(0,07)$ & $0,65 b(0,06)$ & $0,65 b(0,07)$ & $0,66 \mathrm{~b}(0,07)$ \\
\hline C. costaricensis & $0,95 \mathrm{a}(0,02)$ & $0,65 b(0,05)$ & $0,60 \mathrm{~b}(0,05)$ & $0,65 b(0,07)$ & $0,66 b(0,08)$ & $0,60 \mathrm{~b}(0,06)$ \\
\hline Z. longifolia & $0,95 \mathrm{a}(0,01)$ & $0,75 b(0,01)$ & $0,73 b(0,05)$ & $0,65 b(0,06)$ & $0,67 \mathrm{~b}(0,07)$ & $0,59 \mathrm{~b}(0,07)$ \\
\hline Q. insignis & $0,96 a(0,02)$ & $0,66 \mathrm{~b}(0,05)$ & $0,60 \mathrm{~b}(0,07)$ & $0,66 b(0,06)$ & $0,68 \mathrm{~b}(0,02)$ & $0,62 b(0,07)$ \\
\hline N. reticulata & $0,97 \mathrm{a}(0,01)$ & $0,67 \mathrm{~b}(0,05)$ & $0,54 b(0,05)$ & $0,67 b(0,06)$ & $0,65 b(0,07)$ & $0,64 b(0,08)$ \\
\hline C. odorata & $0,95 \mathrm{a}(0,03)$ & $0,65 b(0,06)$ & $0,65 b(0,07)$ & $0,65 b(0,07)$ & $0,67 \mathrm{~b}(0,06)$ & $0,65 b(0,08)$ \\
\hline
\end{tabular}

Letras diferentes muestran diferencias significativas con a 0,05 .

Fuente: Los autores.

de las muestras, la degradación de taninos y ceras de las hojas ocasionan un cambio del color que se puede percibir entre ambas caras.

En el proceso de análisis fotográfico se debe tener el cuidado en la distinción del color del haz y envés de las hojas, (Blagoderov et al., 2012) mencionan que la subjetividad del color entre el haz y el envés es tan relativa a cada especie que en los procesos seriales de fotografiado se tienen que considerar los puntos de referencia para el ajuste del protocolo, siendo recomendado el haz ya que es el menos susceptible a degradación colorimétrica por almacenamiento en el tiempo. (Hutchens, 2011) destaca que, en el proceso de digitalización de las colecciones botánicas, la coloración ideal de referencia debe ser el haz ya que es la parte más susceptible al proceso de secado y almacenado del material.

\section{Efecto del protocolo en la colorimetría de las muestras}

Los análisis realizados mostraron la susceptibilidad del color a la velocidad de obturación, apertura del objetivo y el ISO. Estudios realizados por Hutchens, (2011) en colecciones botánicas encontró que la adaptabilidad de la cámara a las condiciones del sitio y del material es fundamental para la generación de las imágenes representativas del material. El color es el primer indicador que le permite al usuario entender la muestra, si este presenta variaciones significativas puede incidir a un entendimiento erróneo o parcial de la especie. Kao et al. (2018) encontró que la calibración errónea de la cámara puede incidir en cambios radicales del color de órganos o secciones fundamentales de las muestras como son los frutos, flores o texturas en la lámina foliar, generando con ello desconfianza en el material fotografiado y con ello pérdida de su representatividad científica.

Hernández y Navarro (2007) destacan que la sensibilidad de la cámara es fundamental para la calidad de la fotografía, esto debido a que conforme sea mayor el ajuste del color de la muestra, la nitidez y calidad de fotografía podría ser mayor y con ello la muestra tendría una representación acorde al material físico. Wu y Tan (2003) mencionan que la velocidad del obturador es fundamental para la representatividad colorimétrica, en sitios en los que el control luminoso es amplio y que se debe estar fotografiando material estático, el uso de altas velocidades optimiza la fotografía, esto debido que el corto tiempo de captura evita entrada o pérdida de luz por movimientos de elementos cercanos al área de fotografiado, algo que en las velocidades mayores no sea tan simple de controlar; con respecto a la apertura del obturador. 
Vicari, Pisek y Disney (2019) destaca que amplias aperturas en áreas con luminosidad controlada genera aclaramiento de la imagen, lo que significa un aumento de luminosidad y perdida del color. Una apertura grande o muy pequeña implica una pérdida de calidad de la imagen y de nitidez. Thiers et al., (2016) advierte que en herbarios se debe evitar el uso de aperturas superiores a f/13 y menores a $\mathrm{f} / 2$ esto debido a que pueden provocar cambios muy grandes en la calidad del color del material debido a la pérdida de coloración rojiza de las muestras. Finalmente, con el ISO, (Drinkwater et al., (2014) menciona se debe controlar la sensibilidad de captar luz, en condiciones de fotografiado cerrado. En las que la luminosidad sea constante se recomienda utilizar ISO inferiores a 400, esto debido a que valores mayores ocasionan un blanqueamiento de las imágenes, lo cual resulta en una representatividad baja del color del material. Los resultados aquí obtenidos mostraron que valores de ISO menores a 320 fueron los óptimos.

Para los alcances de esta investigación, el protocolo evaluado CP1 presentó mejor adaptabilidad a condiciones expuestas. El control de la luminosidad del sitio en conjunto con la calidad del material es fundamental para una adecuada gestión de la colección digital, Singh y Sharma (2009) destacan tres elementos para la generación fotografías de herbario con calidad colorimétrica: i. con respecto a las coloraciones rojizas en el material, es fundamental se respete el efecto del secado y pérdida de coloraciones verdes de las especies, esto debido al proceso de recoloración que hace necesario la generación de modelos de recuperación colorimétrica. ii. Con respecto a la coloración del material de la lámina, la coloración generalmente blanquecina del material es fundamental para referenciar la manipulación del color, la tendencia es que conforme la muestra tenga mayor edad, la coloración tiende a tornarse amarillenta debido a la oxidación de químicos presentes en papel, una manipulación errónea de la apertura del lente o ISO produciría un aclaramiento atípico del material. iii. pérdida de calidad de la imagen, el ajuste colorimétrico erróneo incide en pérdida del enfoque de la cámara lo que se transforma en disminución del detalle de una muestra.

Con los seis protocolos evaluados únicamente CP1 mostró cumplimiento de los tres principios expuestos, los restantes cinco protocolos mostraron inconsistencias entre especies debido a su incapacidad de adaptación a las distintas condiciones de las muestras. Haston, et al. (2012) menciona que la poca adaptabilidad de los equipos fotográficos a las muestras objetivo y al ambiente se traduce en una gran variación en color y calidad de las imágenes, caso similar al que presentaron los restantes seis tratamientos, por lo que se da una variación acorde a la especie. Magdalena et al. (2018) reitera la necesidad de uniformizar potenciales opciones de ambiente y en el que los usuarios muestran una buena adaptación y entendimiento del material a su equivalente físico, siendo que la claridad y coherencia del color es fundamental en la aceptación de la imagen.

\section{Conclusiones}

Se determinó que es posible generar un protocolo de ajuste fotográfico en el que la colorimetría de las muestras sea representativa a la muestra física. No se encontró un patrón claro en el cambio del color del haz y envés de las hojas de las diez especies, encontrando variaciones significativas entre especies en especial con los valores de luminosidad. De este resultado se desprende la recomendación de utilizar un muestreo del color en el haz debido a que es el lado de la hoja más susceptible al fotografiado. En cuanto a las variables de ajuste del protocolo, en velocidad del obturador las óptimas fueron $1 / 3^{\prime \prime}$ y $1 / 5^{\prime \prime}$ que mostraron $\Delta E^{*}$ inferiores a 6,9 y considerados como perceptibles. Con la apertura del objetivo las mejores fueron $\mathrm{f} / 5.6 \mathrm{y}$ $\mathrm{f} / 7.1$ con $\Delta E^{*}$ de 2,8 a 6,1 y finalmente el ISO ideal obtenido con $\Delta E^{*}$ promedio de 4,8 fueron 100 , 200 y 320. De la mezcla de las distintas opciones de protocolo, se encontró que CP1 fue la única 
que mostró una homogeneidad de resultados con las diez especies y mostró valores promedio de $\Delta E^{*}$ de 3,6 considerados como susceptibles y que en comparación a las restantes mezclas mostró los mejores IRC por parte de los usuarios y una menor variación de Croma, siendo que mostró la mejor adaptación al sitio y condiciones en las que se obtuvieron las fotografías.

\section{Agradecimientos}

Agradecemos al Laboratorio ecofisiología forestal y aplicaciones ecosistémicas (ECOPLANT) y el herbario forestal de la Escuela de Ingeniería Forestal del Tecnológico de Costa Rica, por el apoyo de recursos para desarrollo de la presente investigación

\section{Referencias}

ASTM. (2013). Standard Practice for Calculation of Color Tolerances and Color Differences from Instrumentally Measured Color Coordinates. West Conshohocken, PA: ASTM International.

Barber, A., Lafferty, D., Landrum, L. (2013). The SALIX Method: A semi-automated workflow for herbarium specimen digitization. Taxonomy, 62, 581-590. https://doi.org/10.12705/623.16

Blagoderov, V., Kitching, L., Livermore, L., Simonsen, T., Smith, V. (2012). No specimen left behind: industrial scale digitization of natural history collections. ZooKeys, 209: 133-146. https://doi.org/10.3897/zookeys.209.3178

Cui, W., Kamdem, P., Rypstra, T. (2004). Diffuse reflectance infrared Fourier. Wood and Fiber Science, 36, 291-301.

Drinkwater, R., Cubey, R., Haston, E. (2014). The use of Optical Character Recognition (OCR) in the digitisation of herbarium specimen labels. PhytoKeys, 38, 15-30. https://doi.org/10.3897/ phytokeys.38.7168

Haston, E., Cubey, R., Pullan, M., Atkins, H., Harris, D. (2012). Developing integrated workflows for the digitisation of herbarium specimens using a modular and scalable approach. ZooKeys, 209, 93-102. https://doi. org/10.3897/zookeys.209.3121

Hernández, H., Navarro, M. (2007). A new method to estimate areas of occupancy using herbarium data. Biodiversity and Conservation, 16, 2457-2470. https://doi.org/10.1007/ s10531-006-9134-6

Hutchens, C. (2011). A Look into the Rocky Mountain Herbarium Library Collection and Digital Projects. Serials Review, 37, 35-37. https://doi. org/10.1080/00987913.2011.10765343

Kao, D. H., Djendoel Soejarto, D., Kinghornc, D., Oberlies, D. (2018). Non-destructive chemical analysis of a Garcinia mangostana L. (Mangosteen) herbarium voucher specimen. Phytochemistry Letters, 28, 124-129. https://doi. org/10.1016/j.phytol.2018.10.001

Kovtonyuk, N. H., Gatilova, E. (2017). Digital Herbarium Collections of the Central Siberian Botanical Garden SB RAS, Novosibirsk, Russia. Information Technologies in the Research of Biodiversity, 77, 22-28. https://doi. org/10.1007/978-3-030-11720-7 4

Lang, M., Nilsona, T., Kuuska, A., Piseka, J., Korhonenc, L., Uri, V. (2017). Digital photography for tracking the phenology of an evergreen coniferstand. Agricultural and Forest Meteorology, 246: 15-27. https://doi.org/10.1016/j. agrformet.2017.05.021

Magdalena, U., Estevão Silvaa, L., Oliveira Limaa, R., Belon, R., Ribeiro, F., Oliveiraa, F., . . . Campostrini, R. (2018). A new methodology for the retrieval and evaluation of geographiccoordinates within databases of scientific plant collections. Applied Geography, 96, 11-15. https://doi.org/10.1016/j.apgeog.2018.05.002

Martin, M., Quiroz-Claros, E., Brushb, G., Zimmer, E. (2018). Herbarium collection-based phylogenetics of the ragweeds (Ambrosia,Asteraceae). Molecular Phylogenetics and Evolution, 120, 335-341. https://doi.org/10.1016/j. ympev.2017.12.023

Park, P. (2012). Digital herbarium archives as a spatially extensive, taxonomically discriminate 
phenological record; a comparison to MODIS satellite imagery. International Journal of Biometeorology, 56(6), 1179-1182. https://doi. org/10.1007/s00484-012-0521-2

Riera, R., Sangil, C., Sansón, M. (2015). Long-term herbarium data reveal the decline of a temperate-wateralgae at its southern range. Estuarine, Coastal and Shelf Science, 165, 159-165. https://doi.org/10.1016/j.ecss.2015.05.008

Rivers, C., Taylor, L., Brummitt, N., Meagher, T., Roberts, D., Eimear, E., Lughadh, N. (2011). How many herbarium specimens are needed to detect threatened species? Biological Conservation, 144: 2541-2547. https://doi.org/10.1016/j.biocon.2011.07.014

Shotbolta, L., Bükerb, P., Ashmore, M. (2007). Reconstructing temporal trends in heavy metal deposition: Assessing the value of herbarium moss samples. Environmental Pollution, 147, 120-130. https://doi.org/10.1016/j. envpol.2006.08.031

Singh, S., Sharma, K. (2009). Indian Virtual Herbarium Project: Implementing an institutional knowledge repository as a digital archive - Design, development, solution architecture and implementation. Journal of Digital Asset Management, 5, 55-74. https://doi.org/10.1057/ dam.2008.56

Souza, M., Hawkings, J. (2017). Comparison of Herbarium Label Data and Published MedicinalUse: Herbaria as an Underutilized Source of Ethnobotanical Information. ECOnomic Botany, 1-12. https://doi.org/10.1007/ s12231-017-9367-1

Thiers, b., Tulig, M., Watson, K. (2016). Digitization of The New York Botanical Garden
Herbarium. Brittonia, 68, 324-333. https://doi. org/10.1007/s12228-016-9423-7

Unger, J., Merhof, D., Renner, S. (2016). Computer vision applied to herbarium specimens of German trees: testing the future utility of the millions of herbarium specimen images for automated identification. BMC Evolutionary Biology, 16, 248-255. https://doi.org/10.1186/ $\underline{\text { s12862-016-0827-5 }}$

van der Ent, A., Ocenara, A., Tisserand, R., Sugau, J., Echevarria, G., Erskine, P. (2019). Herbarium X-rayfluorescence screening for nickel, cobalt and manganesehyperaccumulator plants in theflora of Sabah (Malaysia, Borneo Island). Journal of Geochemical Exploration, 202, 49-58. https://doi.org/10.1016/j. gexplo.2019.03.013

Vicari, B., Pisek, J., Disney, M. (2019). New estimates of leaf angle distribution from terrestrial LiDAR: Comparisonwith measured and modelled estimates from nine broadleaf tree species. Agricultural and Forest Meteorology, 264, 322-333. https://doi.org/10.1016/j. agrformet.2018.10.021

Willis, ,., Ellwood, E., Primack, R., Davis, C., Pearson, K., Gallinat, A., . . . Soltis, P. (2017). Old Plants, New Tricks: Phenological Research Using Herbarium Specimens. Trends in Ecology and Evolution, 32, 531-546. https://doi.org/10.1016/j.tree.2017.03.015

Wu, S., Tan, B. (2003). Valuable and Noteworthy Chinese Fern Specimens Preserved at the Herbarium of Singapore Botanic Gardens (Sing). Pteridology in the New Millennium, 144-159. https://doi. org/10.1007/978-94-017-2811-9 12 